\title{
Percepção dos enfermeiros sobre desenvolvimento de competências durante a residência
}

\author{
Perception of nurses on developing skills during residency
}

Percepcion de las enfermeras em el desarrollo de habilidades durante la residencia

Maria do Socorro Batista Sena Leite ${ }^{1}$, Carmina Silva dos Santos ${ }^{1}$, Thálita Cavalcanti Menezes da Silva ${ }^{1}$, Jerônimo Faustino do Rego Filho ${ }^{1 *}$, Liana Chaves Alves', Kelly Cristina Torres Lemes ${ }^{1}$.

\section{RESUMO}

Objetivo: Analisar a percepção dos residentes sobre o desenvolvimento de competências necessárias à qualificação dos enfermeiros durante o Programa de Residência em Enfermagem de um hospital do estado de Pernambuco. Métodos: Tratou-se de uma pesquisa qualitativa, utilizando o método de análise temática de conteúdo de Bardin FB (1977); cujos dados foram coletados junto a seis residentes mediante grupo focal. A pesquisa foi aprovada pelo Comitê de Ética da Faculdade Pernambucana de Saúde e a coleta de dados foi realizada no período de julho a agosto do ano de 2014. Resultados: Mediante análise temática o estudo identificou nove aspectos categóricos, que possibilitou sistematizar os resultados em dois temas: Potencialidades e Fragilidades do Programa de Residência de Enfermagem. Conclusão: As potencialidades do programa foram: a relevância do curso para a capacitação dos residentes, que possibilita 0 desenvolvimento de competências necessárias para a qualificação profissional, agregando a teoria à prática, o campo oferecido é muito amplo que favorecem a formação profissional dos residentes do programa de Residência de Enfermagem no agreste de Pernambuco. E como fragilidades: falta apoio institucional, melhor definição do papel do residente na instituição, falta de capacitação dos preceptores.

Palavras chave: Qualificação profissional, Competências, Residência.

\begin{abstract}
Objective: To understand the perceptions of residents about the development of necessary skills the qualification of nurses during the Residency Program in Nursing of a hospital in the state of Pernambuco. Methods: This was a qualitative study using thematic analysis method content Bardin FB (1977); with data collected by the six residents through focus group. The research was approved by the Ethics Committee of Pernambuco Faculty of Health and data collection was carried out between July-August of 2014. Results: Through thematic analysis study identified nine categorical aspects, which made it possible to systematize the results on two themes: Potential and Weaknesses of Nursing Residency Program. Conclusion: The program's potential were: the relevance of the course for the training of residents, which enables the development of skills required for vocational training, adding theory to practice, the course offered is very broad favoring the qualification of residents Nursing Residency Program in the wild of Pernambuco. And as weaknesses: lack institutional support, better definition of resident's role in the institution, lack of training of tutors.
\end{abstract}

Keywords: Professional qualification, Skills, Residence.

\section{RESUMEN}

Objetivo: Analizar las percepciones de los residentes en el desarrollo de las habilidades necesarias para La qualificación de personal de enfermería durante el Programa de Residencia em Enfermería del de un hospital

${ }_{1}^{1}$ Faculdade Pernambucana de Saúde (FPS), Recife - PE. *E-mail: jeronimofausto@yahoo.com.br SUBMETIDO EM: 6/2020 ACEITO EM: 7/2020 PUBLICADO EM: 10/2020 
em el estado de Pernambuco. Métodos: Se realizo un estúdio cualitativo utilizando el método de análisis temático de contenido de Bardin FB (1977); con los datos recogidos por los seis residentes a través de grupos focales. La investigación fue aprobada por el Comité de Ética de Pernambuco Facultad de Salud y la recolección de datos se llevó a cabo entre julio y agosto de 2014. Resultados: Através Del estudio de análisis temático identificaron nueve aspectos categóricos, que permitió sistematizar los resultados en dos temas: Potencial y Debilidades del Programa de Residencia Enfermería. Conclusión: El potencial del programa fueron: la pertinencia del curso para La qualificación de residentes, lo que permite El desarrollo de las habilidades necesarias para La qualificación profesional, La adición de La teoría a La práctica, el curso se ofrece es muy amplia a favor de la formación de los residentes Programa de Residencia de Enfermería em La naturaleza de Pernambuco. Y como debilidades: falta de apoyo institucional, una mejor definición del papel del residente em La institución, la falta de formación de tutores.

Palabras clave: Qualificación profesional, Habilidades, Residencia.

\section{INTRODUÇÃO}

Os Programas de Residência de Enfermagem visam desenvolver profissionais competentes e qualificados para o desenvolvimento de habilidades práticas, com vistas a sua inserção nos serviços de saúde, onde proporcionarão uma assistência pautada nos princípios do Sistema Único de Saúde (SUS). Utilizando os saberes adquiridos na graduação, com a aplicação nos campos de pratica profissional, o enfermeiro em formação e inseridos no contexto social dos usuários, desenvolve uma capacidade de promover uma integralização da relação teoria e prática no seu exercício profissional, de modo a prestar uma assistência de saúde adequada à população (BOUSSO RS, et al., 2000; ITO EE, TAKAHASHI RT, 2005; OLIVEIRA MAC, 2009).

Neste sentido, os profissionais, durante o processo da residência, adquirem competências com vistas à integralidade da assistência, a qual requer das instituições formadoras a implantação de ações de mudanças, capazes de assegurar a reorientação dos processos de formação, que prepare os profissionais para as práticas que contemplem ações de promoção, proteção, prevenção, atenção precoce, cura e reabilitação (GIL CRR, 2005). Dessa forma a competência clínica pode ser entendida como a capacidade profissional de realizar ações diretamente relacionadas aos cuidados dos pacientes. Consiste, portanto na capacidade de agir em determinada situação, baseado nos conhecimentos cognitivos e afetivos para interagir em situações complexas, mobilizando e aplicando recursos, por meio da ação profissional (LIMA VV, 2005; PERRENOUD $\mathrm{PH}, 2002)$.

Neste contexto, os espaços de prática e os fenômenos vivenciados pelos profissionais funcionam como estratégias pedagógicas que, durante a residência, permite integrar o processo ensino-aprendizagem, no qual as atividades práticas dos residentes estão relacionadas ao treinamento em serviço, de acordo com as especialidades das áreas de concentração, sob a supervisão de um docente e um preceptor, onde o primeiro tem a função de transmitir o conhecimento e o segundo a função de supervisão direta das atividades práticas dos residentes (BRASIL, 2014).

Foi diante dessa percepção que se justificou a necessidade de estudos nessa área, motivando a proposição dessa pesquisa, que teve como objetivo analisar a percepção dos residentes sobre o desenvolvimento de competências necessárias a formação durante a residência de enfermagem de um hospital do estado de Pernambuco.

\section{MÉTODOS}

Estudo descritivo, exploratório, com abordagem qualitativa. A pesquisa de campo foi realizada no de um hospital do estado de Pernambuco, tendo como sujeitos de pesquisa, os enfermeiros residentes, inseridos no Programa de Residência de Enfermagem do referido Hospital.

A coleta de dados se deu entre os meses de julho a agosto de 2014, obedecendo aos seguintes critérios de elegibilidade dos sujeitos: residentes do primeiro e segundo ano do Programa de Residência de 
Enfermagem (PRENF) que, no período da pesquisa, estivessem desempenhando atividades assistenciais, nos setores das especialidades das Clínicas Cirúrgicas, Emergência e Unidade de Terapia Intensiva (UTI).

A coleta de dados foi realizada por meio de dois encontros de grupo focal com os enfermeiros residentes do PRENF. Os dados obtidos foram gravados com a devida anuência dos participantes, transcrito na íntegra e para o entendimento dos significados das falas, foi utilizada a análise de conteúdo temática Bardin FB (1977).

Adotou-se uma codificação das representações, através da categorização, interpretação e o desenvolvimento de explicações e representações dos participantes, com vista ao alcance dos objetivos propostos na pesquisa.

Foram realizados dois encontros, com o mesmo grupo, composto por nove componentes: sendo seis residentes, a pesquisadora que, no papel de moderadora, contou com uma auxiliar e uma observadora. No tratamento dos dados os residentes receberam os codinomes de: $R_{1}, R_{2}, R_{3}, R_{4}, R_{5}, R_{6}$. Respectivamente para garantir anonimato. Os encontros foram realizados na biblioteca do referido hospital, com duração de uma hora e trinta minutos cada, os diálogos foram gravados por meio digital, para posterior transcrição e análise das falas. Durante a operacionalização foram apresentadas questões norteadoras para que os participantes emitissem seus depoimentos e impressões.

Durante as reuniões, foram respeitadas as diretrizes da Resolução do Conselho Nacional de Saúde de no 466 de dezembro de 2012. O estudo foi apreciado pelo Comitê de Ética da Faculdade Pernambucana de Saúde (FPS), conforme Parecer Consubstanciado oㅜ 714.755 e CAAE oㅜ 32004914.1.0000.5569. Os participantes que aceitaram voluntariamente, participar do estudo, assinaram o Termo de Consentimento Livre e Esclarecido (TCLE).

\section{RESULTADOS E DISCUSSÃO}

Foram levantados os seguintes dados: A idade dos residentes varia entre 23 e 26 anos, todas do sexo feminino, com tempo de conclusão de formação que variou entre dez meses a dois anos e sete meses. As residentes estavam distribuídas nas Clínicas Cirúrgicas, Emergência e Unidade de Terapia Intensiva (UTI), áreas do programa. Corroborando com achado dos autores (REGO FILHO JF, SANTOS CS, 2017; REGO FILHO JF, et al., 2020).

Leitura e Interpretação dos dados: para realizar a análise temática dos dados foram destacadas em cada codinome as unidades de registro, tendo sido enumeradas cento e duas (102) unidades de registros, as quais foram separadas por idéias e grifadas os núcleos de sentido dos depoimentos, destacando as palavras importantes que tiveram significado para os objetivos da análise, daí a formulação das categorias, que segundo Bardin FB (1977), para realizar a análise categorial, é necessário destacarem os termos comuns, e fazer o agrupamento e a classificação dos elementos.

Mediante essa análise temática o estudo possibilitou identificar os seguintes aspectos categoriais apresentadas abaixo: Categoria 1: Prática - importante para a formação e desenvolvimento de competências; Categoria 2: Habilidades - aprendizado desenvolvido durante a vida acadêmica, as quais capacita o profissional para a prática, mas que nem sempre são necessariamente desenvolvidas; Categoria 3: Funções institucionais do residente; Categoria 4: Teoria à prática em suas possibilidades de agregação; Categoria 5: Diferença entre Residência e Especialização; Categoria 6: Apoio institucional; Categoria 7: A preceptoria; Categoria 8: Competências desenvolvidas durante o programa; Categoria 9: Contribuição do programa de residência para a formação profissional. Esses aspectos que tratamos como categorias indicadoras de análise foram agrupadas em dois temas: Potencialidades e Fragilidades do Programa de Residência de Enfermagem.

\section{Tema 1 - Potencialidades do Programa de Residência de Enfermagem do HRA (PRENF) no desenvolvimento de competências}

Dos nove aspectos categóricos agrupados, seis se enquadraram como potencialidades do programa, conforme relacionados abaixo. 
Quanto a Prática como fator importante para a formação e desenvolvimento de competências, a residência de enfermagem se configura como um período de prática ímpar no processo de formação dos profissionais. Corroborando com os depoimentos do grupo essas habilidades práticas podem ser demonstradas nas seguintes falas, abaixo:

[...] Pra mim a residência é a oportunidade de poder desenvolver as minhas qualidades, tanto técnicas quanto teóricas, no caso técnica-científicas, porque a residência é a especialização voltada mais para a prática. $\left(R_{4}\right)$;

[...] A prática no Hospital Regional do Agreste é muito rica, pois, por melhor que seja a Universidade, ela deixa muito a desejar em relação à prática. $\left(R_{1}\right)$;

[...] O hospital é um campo muito amplo, acaba nos aprimorando quanto à parte prática em si e agregando também a parte teórica. $\left(R_{3}\right)$;

[...] Nossa visão científica agrega também àquela prática que o setor nos oferece. $\left(R_{5}\right)$

Esses relatos corroboram com a literatura quando autores afirmam que a residência é uma modalidade de pós-graduação que capacita o profissional enfermeiro para a aquisição de experiência e maturidade necessária ao desempenho profissional, estabelecendo uma ponte entre a teoria e a prática (SHIVARDI SN, 1997). Propiciando a proficiência necessária que o mercado de trabalho exige integrando os aspectos assistenciais, administrativos, de ensino e pesquisa, sob supervisão (CANNATO FGA, 1999).

Quanto à Possibilidade de agregar teoria à prática, a residência tem se constituído como um espaço de prática que possibilita desenvolver as competências, que não foram possíveis de serem desenvolvidas na graduação. Conforme é demonstrado nas falas abaixo:

[...] O programa vai me trazer uma unificação prática, um desenvolvimento prático, um aprimoramento prático, mas agregado ao valor teórico. Fazendo a troca desses conhecimentos $\left(R_{6}\right)$;

[...] A residência vai trazer principalmente um amadurecimento profissional. São competências técnicas: vai criar habilidades de liderar, coordenar grupo, uma equipe (R4);

[...] Ela ajuda a desenvolver as minhas competências $\left(R_{5}\right)$;

[...] Vai agregar habilidades técnicas e teóricas na residência $\left(R_{3}\right)$.

Esse processo consiste segundo as falas das entrevistadas em momento de desenvolvimento de competências, que segundo a conceituação, é abordada a partir de vários ângulos: desenvolvimento de competências consiste em mobilizar conhecimentos, porque não existem regras prontas, é necessário desenvolver o senso crítico e criativo para enfrentar determinadas situações (PERRENEUD PH, 1999).

É necessário ressaltar que as instituições formadoras e seus formadores enfrentam os desafios de desenvolver profissionais competentes, para atuarem no mundo globalizado, com as novas tecnologias e novas formas de trabalho, para atender aos princípios do SUS (PERRENOUD PH, 2002). Sendo assim as competências dos profissionais de saúde são adquiridas e construídas, através das oportunidades que os serviços oferecem, para que estes desenvolvam suas habilidades e adquiram experiências, com as vivências do dia a dia (SILVA MG, et al., 2010).

Quanto a Competências desenvolvidas durante o PRENF, no presente estudo, as residentes consideraram que as ações desenvolvidas no ambiente hospitalar, contribui para a sua formação profissional. Conforme é demonstrado nas falas:

[...] Esse programa me permite um amadurecimento profissional, porque a partir dele eu vou criar manejo e desenvolver forma de lidar com outros profissionais da área $\left(R_{1}\right)$; 
[...] Na experiência você vai criar habilidades para liderar, para coordenar um grupo, uma equipe, para poder realizar ações decisivas em momentos oportunos, enfim, é competência técnica, que vai lhe deixar mais amadurecido e mais profissional $\left(R_{4}\right)$;

[...] Nós preparamos os seminários, ministramos essa parte teórica e é válido, porque agente vai se aprofundar e acaba se aprimorando $\left(R_{3}\right)$;

[...] O residente tem a função de aperfeiçoar o serviço onde está alocado $\left(R_{2}\right)$;

[...] A gente proporciona capacitação pra os profissionais do hospital todo ano, fazendo palestras sobre queimados no período junino $\left(R_{5}\right)$.

[...] A gente consegue fazer uma ou duas vezes por semana a visita clínica, uma passagem de plantão mais detalhada e consegue discutir o caso do paciente $\left(R_{6}\right)$.

O hospital oferece um campo muito rico e amplo no aperfeiçoamento prático, onde se espera que o residente tenha competências gerenciais em sua prática assistencial, tais como: relacionamento interpessoal, liderança, competência técnica, tomada de decisões e compromisso (HELLER A, 1994).

É da compreensão geral das unidades de formação acadêmica que tanto o processo de graduação como as formas de especializações posteriores constituem momentos de desenvolvimento de competência para o exercício profissional. A residência também se insere nesse processo de formação, com o objetivo de aprimorar competências, de modo a assegurar uma melhor qualidade do fazer profissional (SILVA MG, et al., 2010).

Quanto a Diferença entre a especialização e a residência de enfermagem, nesse aspecto de análises o grupo levantou questões relacionadas à diferença entre os dois tipos de modalidades de pós-graduação latosensu: a especialização e a residência em enfermagem: Essa concepção está explicitada conforme as falas abaixo:

[...] Na residência você tem que ir buscar a teoria e aplicar ali na pratica e na especialização o instrutor dá a teoria, mas fica algo vago, porque você não vai aplicar simultaneamente. $\left(R_{5}\right)$;

[...] A diferença entre a residência e as outras especializações, é que se você quer formar um especialista, você tem que formar alguém que além de ter o conhecimento científico, ele tem que ter também a habilidade e nós achamos que essa habilidade, ela só vai ser adquirida com a residência. $\left(R_{2}\right)$.

Neste sentido, a diferença entre a especialização e a residência é justamente esta relação da teoria com a prática, onde na residência evidencia uma capacitação mais ampla para o profissional, funciona como uma ponte entre a teoria e a prática (LOPES GT, MOURA CFS, 2004). Além disso, propicia ao enfermeiro recémgraduado a maturidade e a experiência prática necessária para o ingresso no mercado de trabalho, sendo 0 fator facilitador no processo de formação (GARCIA LAMG, 2005).

Quanto a Contribuição do programa de residência para a formação profissional, ainda que apresente limitações, o Programa de Residência oferece as possibilidades de formação, dentro de determinadas condições, inerentes aos recursos destinados ao programa, assim como as condições de funcionamento institucionais do hospital. Conforme as falas abaixo:

[...] A contribuição principal é o campo oferecido, que tem recursos humanos e materiais, além de profissionais dispostos a tirar dúvidas. Oferece insumos e recursos, como por exemplo: coberturas especiais para serem utilizadas em curativos $\left(R_{4}\right)$;

[...] Um dos exemplos são os insumos e equipamentos modernos como: bombas de infusão, cateteres, curativos a vácuo, enfim são oportunidades que a gente tem. Porque a contribuição mesmo é a área que a residente precisa para aprender $\left(R_{5}\right)$; 
[...] O cenário de pratica te dá tudo o que você precisa em relação a pacientes com tal patologia você tem ali, se precisa de tal cobertura para curativo você tem. O que falta mesmo é um preceptor presente para orientar $\left(R_{3}\right)$;

[...] O campo de prática é muito rico, são tantos procedimentos que são ricos para a minha formação $\left(R_{6}\right)$.

Ainda em relação aos cenários de prática, os depoimentos revelam que:

[...] Na experiência da emergência, sem dúvida é um campo muito rico, porque a gente vê uma infinidade de casos clínicos onde podemos vivenciar diversas situações $\left(R_{2}\right)$;

[...] A UTI possui uma estrutura adequada, que proporciona um conhecimento maior $\left(R_{4}\right)$.

Esses achados corroboram com a literatura, quando os estudos sobre a residência de enfermagem, tem sido vista por alguns autores, como uma forma de proporcionar qualificação profissional aos enfermeiros recém-graduados (CYLINDRO AC, 2000).

Alguns comparecem porque têm interesse em atualizarem-se, mas a maioria vem pela qualificação e obtenção de certificados, para comprovação da carga horária, no recebimento da produtividade. Tudo isso incorre também na pouca divulgação e participação também nos seminários. Conforme afirma as falas:

[...] Eu acho que eles só passaram a participar desses cursos depois que foi implantada essa questão de gratificação por produtividade $\left(R_{2}\right)$;

[...] Sentimo-nos desestimuladas pelo fato de sabermos, que só estão ali pelo benefício da carga horária, não pela capacitação $\left(R_{1}\right)$;

[...] É o que sempre as preceptoras falam nos nossos seminários e muitas vezes os profissionais não tomam nem conhecimento. Mas oque falta é isso mesmo, só divulgação $\left(R_{6}\right)$.

Durante a vida acadêmica não há desenvolvimento de habilidades, os residentes apesar de terem os conhecimentos adquiridos, sentem dificuldades em relacionar os conteúdos aprendidos, com a realidade apresentada, porque as experiências dos estágios durante a graduação é insuficiente em relação à prática, conforme é demonstrado nas falas a seguir:

[...] As ações vêm contribuir com a nossa formação durante a vida acadêmica, mas falta o desenvolvimento de algumas habilidades que deveríamos ter, e a residência proporciona isso $\left(R_{6}\right)$;

[...] Nossa visão científica vem para agregar àquela prática que Setor tá oferecendo a gente $\left(R_{5}\right)$;

[...] A gente tem oportunidade de desenvolver vários procedimentos, que na vida acadêmica, agente não havia realizado $\left(R_{2}\right)$;

\section{[...] A universidade deixa a desejar em relação à prática $\left(R_{1}\right)$.}

Os enfermeiros escolhem o curso nos moldes da residência de enfermagem, porque o curso oferece um perfil de formação teórica mais complexa onde os profissionais buscam a informação e por permitir as trocas de experiências entre os profissionais e professores na área, para possível compensação das deficiências da graduação em relação à prática, além de capacitar para o mercado de trabalho (AGUIAR BCG, et al., 2004).

Mesmo com as mudanças curriculares dos cursos de saúde entre eles a enfermagem, para atender aos princípios e condições das novas Diretrizes Curriculares Nacionais (LDB), onde estabelece a necessidade de uma maior integralização do processo teórico-prática, levando a formação em enfermagem centrada numa contínua aproximação do mundo do ensino com o mundo do trabalho (FERNANDES JD, et al., 2005). 


\section{Tema 2 - Fragilidades do Programa de Residência de Enfermagem}

Dos nove aspectos categóricos encontrados, três destes se configuraram nas fragilidades do programa, conforme está apresentado abaixo:

Quanto ao papel/ lugar ocupado pelo residente na instituição, o papel das residentes é confundido com função da enfermagem contratada e as atividades da residência não se diferenciam das ações cotidianas do exercício das contratadas. Assim, a residência parece ser vista dentro da instituição como força de trabalho e não como atividade de qualificação. Diante disso que as residentes sentem a necessidade de maiores informações para esclarecer aos demais profissionais o papel do residente no serviço. Conforme é demonstrado nas falas:

[...] Os profissionais que ali estão, acabam por não saber o que é um residente, pra que se propõem os residentes $\left(R_{4}\right)$;

[...] Tem gente que acha que somos estagiarias, tem gente que acha que não somos formadas, que nós somos acadêmicas $\left(R_{1}\right)$;

[...] Acho que as pessoas deveriam entender o que é uma residência, pra saber identificar o papel deste, ao invés de só cobrar, cobrar $\left(R_{2}\right)$;

\section{[...] Divulgar o que é a residência, qual o papel do residente $\left(R_{6}\right)$.}

De acordo com a literatura, o enfermeiro residente durante o período da residência, se encontra num "rito de passagem", porque fica dividido como aluno de pós-graduação, quando está na instituição de ensino e de profissional de enfermagem quando está nos cenários de prática. Portanto quando está em treinamento em serviço, o residente desempenha a função de enfermeiro (AGUIAR BCG, et al., 2004).

Quanto ao Apoio Institucional, os residentes não se sentem reconhecidas e valorizadas pelos seus esforços no desempenho dessas atividades. Isto é demonstrado nas falas:

[...] Nós também trazemos benefício para o serviço e muitas vezes nos dispomos a fazer palestras, minicursos. A gente aguarda o retorno do hospital, que muitas vezes não tem $\left(R_{6}\right)$;

[...] Existem profissionais do programa que fazem parte da coordenação que tem dado apoio à residência, mas não recebem nenhum incentivo para estar ali. Então eles fazem porque eles querem de alguma forma ver o retorno, tanto para nossa formação, como para o hospital $\left(R_{5}\right)$;

[...] Então os profissionais que estão, acabam por não saber o que é um residente, a que se propõe. Pensam que é só mão-de-obra. Então, nesse caso, eu acho o apoio da instituição deficiente $\left(R_{4}\right)$.

Quanto a Preceptoria, do conjunto de atividades dos residentes de enfermagem do HRA, nos setores onde estão alocados, com relação aos enfermeiros assistenciais, que também são preceptores do programa de residência, constitui o espaço onde deveria haver a troca de experiências e conhecimentos, entretanto, isso nem sempre ocorre. Conforme as falas:

[...] A residência vem mais com a parte teórica e deveria haver essa troca entre $o$ enfermeiro do setor que tem a parte prática, o que muitas vezes não ocorre $\left(R_{2}\right)$;

[...] A gente vai poder aplicar o conhecimento e de acordo com o enfermeiro do setor, poderemos fazer a troca desses conhecimentos, ou seja, trocando experiências $\left(R_{6}\right)$;

\section{[...] Está muito solta à relação entre a residência e a enfermagem do $H R A\left(R_{4}\right)$.}

A ausência desse intercâmbio entre os conhecimentos teóricos e a experiência prática, pode vir a resultar em uma dicotomia entre o pensamento e a realidade do dia a dia pelos profissionais, de modo que fragiliza o processo de capacitação e crescimento profissional, que poderia ocorrer em mão dupla: os enfermeiros 
repassando as habilidades e experiências para os residentes e estes que por sua vez compreenderia a forma de aplicação dos seus conhecimentos na prática. Essa situação está explicito nas falas:

[...] A nossa maior defasagem mesmo seria na prática, a gente não tem aquele preceptor com tempo disponível para discutir o caso clinico apesar deles tentarem fazer mesmo assim, o que podem, entre plantões e plantões, que eles têm que assumir, além de outras atividades $\left(R_{3}\right)$;

[...] É tudo o que está problematizando a residência, pois é uma cadeia, não tem isso, não tem aquilo, acaba não tendo muitas coisas $\left(R_{4}\right)$.

Nesse sentido, a contribuição do enfermeiro assistencial, que também é preceptor do programa, é necessária e importante na prática para acompanhar e ensinar aos residentes, mas que lhe são dadas tantas atribuições burocráticas e assistenciais, que não se sentem estimulados para o ensino. Daí advir à necessidade de uma capacitação pedagógica para os preceptores (LOPES GT, MOURA CFS, 2004).

\section{CONCLUSÃO}

Constatou-se a relevância do curso para a capacitação profissional dos residentes de enfermagem que dispõe de recursos humanos, insumos, equipamentos modernos e uma grande variedade de procedimentos, agregando tanto a parte prática quanto a teórica. Como também a falta de uma capacitação pedagógica para os enfermeiros assistenciais, uma vez que também são preceptores do programa. Acreditam que contribuem para melhorar a assistência da enfermagem nos setores do hospital, sentiram a falta de apoio institucional para melhor qualificar-se. Mas por outro lado o campo é muito rico e favorece o desenvolvimento de competências necessárias à qualificação profissional.

\section{REFERÊNCIAS}

1. AGUIAR BGC, et al. Especialização nos moldes da residência de Enfermagem. Rev. Bras. Enfermagem, 2004; 57(5): 555-559.

2. $\quad$ BARDIN FB. Análise de Conteúdo. Lisboa. Portugal: 1977; Edições 70.

3. BRASIL. Lei no 11.129, de 30 de junho de 2005; institui o Programa Nacional de Inclusão de Jovens - Pro-jovem; cria o Conselho Nacional da Juventude- CNJ. E a Secretaria Nacional de Juventude; altera as leis nos 10.683 de 28 de maio de 2003 e 10.429 de 24 de abril de 2002. Brasília (DF)2005-2007. Disponível: http//www.defesa.gov.br/saude/L11129.pdf. Acesso: julho/2014.

4. BOUSSO RS, et. al. Estágio curricular em enfermagem: transição de identidades. REEUSP. 2000;34(2):218-25

5. CANNATO FGA. O Residente de Enfermagem e o Mercado de Trabalho: Expectativas e Possibilidades Concretas, RJ. Dissertação (Mestrado em Enfermagem) Escola Anna Nery. Universidade Federal do Rio de Janeiro, Rio de Janeiro, 1999; 83 p.

6. CYLINDRO AC, et al. Residência de Enfermagem: uma trajetória de 20 anos. Rev. bras. enferm, 2000; 53(21):109-114.

7. De MELO ARFF, et al. Concepção dos enfermeiros na estratégia saúde da família referente a uma capacitação problematizadora em um município do estado Pernambuco. Revista Eletrônica Acervo Saúde, 2020; (44): 1-8p.

8. FERNANDES JD, et al. Diretrizes Curriculares e Estratégias para Implantação de uma Nova Proposta Pedagógica. Rev. ESc. De Enfermagem, USP, 2005; 39(3): 443-9.

9. GARCIA LAMG. Competências e Habilidades: você sabe lidar com isso? Educação e Ciência On-line. Brasília: Universidade de Brasília. Disponível em: http://uvnt.universidadevirtual.br/ciências/002.htm. Acesso em: 17/02/2015

10. GIL CRR. Formação de recursos humanos em saúde da família: paradoxos e perspectivas. Cad. Saúde Pública, 2005; 21(3): 490498.

11. HELLER A. Sociologia de La vida cotidiana. 4ª Ed. Barcelona: Ediciones Península. 1994; 432p.

12. ITO EE, Takahashi RT. Percepção dos enfermeiros de campo sobre o estágio curricular da graduação de enfermagem realizados em sua unidade de trabalho. REEUSP. 2005; 39(1):109-10.

13. LIMA VV. Competência: distintas abordagens e implicações na formação de profissionais de saúde. Interface - Comum Saúde Educ. 2005; 9(17): 369-79.

14. LOPES GT, MOURA CFS. O impacto da residência de Enfermagem na reconfiguração do enfermeiro assistencial: 1975-2000. Esc. Anna Nery. rev. bras. enferm, 2004; 8(2): 39-45.

15. OLIVEIRA MAC. Da Intenção ao Gesto: a Dialética da Formação de Enfermagem em Saúde Coletiva, SP. Tese (Doutorado em Enfermagem)__Escola de Enfermagem. Universidade de São Paulo, São Paulo, 2004; 89p.

16. PERRENOUD PH. Construir competências desde a escola. $2^{2}$ Ed. Porto Alegre. Artmed. 1999.

17. PERRENOUD PH. A prática reflexiva no ofício de professor: profissionalização e razão pedagógica. Porto Alegre: Artmed Editora. 2002.

18. REGO FILHO e SILVA CS. Residência em enfermagem: identificação das atividades de preceptoria em um Hospital escola. O Mundo da Saúde, São Paulo, 2018; 42(2): 333-348.

19. SHIVARDI SN. Análise da Implantação de um Curso de Especialização, Mod. De Residência de Enfermagem, em uma Instituição de Ensino Superior Privada. SP. Dissertação (Mestrado em Enfermagem) _Universidade Mackenzie, São Paulo, 1977, 97p.

20. SILVA MG, et al. Processo de Formação do Enfermeiro na Contemporaneidade: Desafios e Perspectivas, Texto, Contexto Enfermagem, 2010; 19(1): 176-84. 